\title{
Combination of Extracorporeal Shockwave Lithotripsy and Ureteroscopy for Large Staghorn Calculi in a Pediatric Patient: Case Report
}

\author{
Austin J. Schults, BA, Wanjian Jia, MD, PhD, Michael C. Ost, MD, ${ }^{1}$ and Siam Oottamasathien, MD ${ }^{1}$
}

\begin{abstract}
Background: Extracorporeal shockwave lithotripsy (SWL) is a procedure commonly performed to treat nephrolithiasis, with promising results in pediatric patients. However, increasing renal calculi size is directly related to worsening stone-free rates. There are few reports in the literature of $>2-\mathrm{cm}$ staghorn calculi that expound on the exact mechanism of treatment in the pediatric population.

Case Presentation: We present a case report of a 3-year-old boy who presented with a large 3-cm staghorn calculi effectively treated with one session of SWL followed by a planned staged ureteroscopy for definitive treatment.

Conclusion: Despite the numerous studies limiting the use of SWL to treat stones $<2 \mathrm{~cm}$, if used in softer composition stones, coupled with the larger focal volume involved with smaller patients, SWL when used in combination with adjunctive ureteroscopy is a safe and effective treatment option.
\end{abstract}

Keywords: staghorn, urinary calculi, nephrolithiasis, extracorporeal shockwave lithotripsy

\section{Introduction}

T HE INCIDENCE OF PEDIATRIC UROLITHIASIS of the upper urinary tract is increasing. ${ }^{1}$ One widely accepted procedure for treating renal calculi is extracorporeal shockwave lithotripsy (SWL). Although a well-tolerated procedure in pediatric patients, current stone-free rates with SWL are difficult to interpret from the existing body of data due to discrepancies between study parameters. We report a rare case of a 3-year-old boy who received SWL to treat a struvite staghorn calculus effectively.

\section{Case Presentation}

A 3-year-old boy presented with gross painless hematuria. Diagnostic plain-film imaging was performed revealing a large $3-\mathrm{cm}$ left-sided renal pelvis staghorn calculus with extension into the interpolar and lower pole calices. There also appeared to be a separate stone in the upper pole calix measuring $\sim 1 \mathrm{~cm}$ (Fig. 1a). A renal ultrasound was also performed demonstrating the left kidney to have healthy parenchyma with no evidence of hydronephrosis (Fig. 1b). Multiple hyperechoic structures filling the renal pelvis and calices with acoustic shadowing were clearly appreciable. We felt this diagnostic workup was thoroughly sufficient and did not feel that imaging with a CT scan was necessary, minimizing overall radiation exposure. The patient also had a positive urine culture for Proteus mirabilis, treated appropriately, and kept on bactrim prophylaxis until the day of surgery. His presentation was consistent with a magnesium ammonium phosphate (MAP) or struvite stone. The patient was otherwise healthy with no other medical problems.

Traditionally, struvite calculi are less dense in composition, coupled with a large focal volume for SWL in a 3-year old; the choice was made to pursue SWL as the first-line treatment modality. Initially, we performed cystoscopy with a left retrograde ureteral pyelogram, and a Double-J ureteral stent was placed without difficulty. The patient's lungs were shielded to prevent pulmonary contusions. We then

\footnotetext{
${ }^{1}$ Division of Pediatric Urology, Department of Surgery, Primary Children's Hospital, University of Utah, Salt Lake City, Utah.

${ }^{2}$ Division of Pediatric Urology, Children's Hospital of Pittsburgh, University of Pittsburgh Medical Center, Pittsburgh, Pennsylvania.

(C) Austin J. Schults et al. 2017; Published by Mary Ann Liebert, Inc. This is an Open Access article distributed under the terms of the Creative Commons Attribution License, which permits unrestricted use, distribution, and reproduction in any medium, provided the original work is properly cited. Mary Ann Liebert, Inc. offers reprint services for those who want to order professionally produced copies of articles published under the Creative Commons Attribution (CC BY) license. To obtain a price quote, email Reprints@liebertpub.com. Please include the article's title or DOI, quantity, and delivery destination in your email.
} 

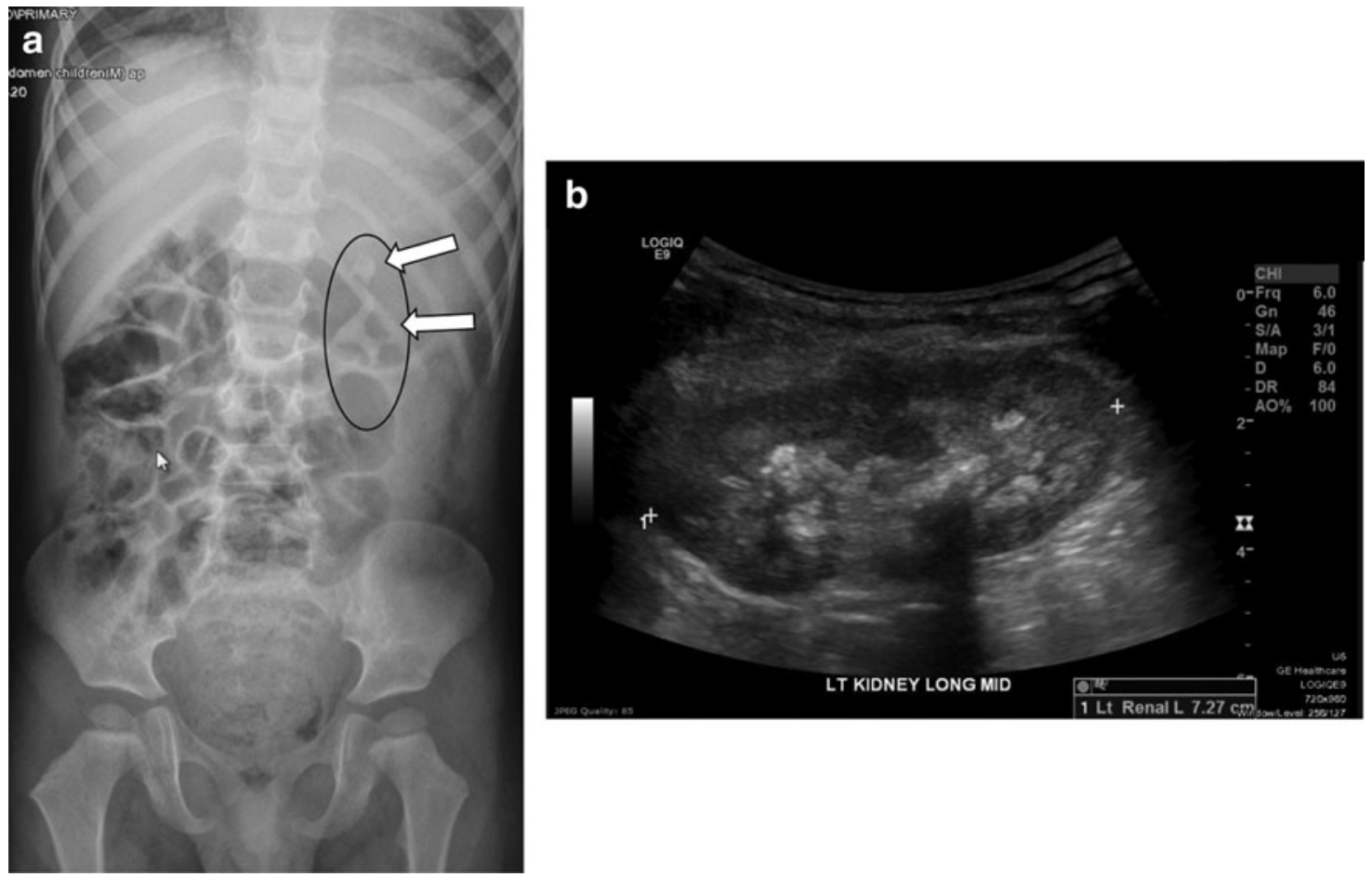

FIG. 1. (a) Diagnostic plain film imaging revealing a large 3-cm left-sided renal pelvis staghorn calculus with extension into the interpolar and lower pole calices, with another separate stone in the upper pole calix measuring $\sim 1 \mathrm{~cm}$. (b) A renal ultrasound demonstrating the left kidney to have healthy parenchyma with no evidence of hydronephrosis. Multiple hyperechoic structures filling the renal pelvis and calices with acoustic shadowing are clearly appreciable.

performed one session of SWL at a low rate of $60 \mathrm{~Hz}$ and a power of 5 using a Storz/GE OEC 9800 lithotripter. The patient received 2000 shocks to the main staghorn calculus at its extension from the renal pelvis into the interpolar and lower pole regions. An additional 500 shocks were then applied to the upper pole $1-\mathrm{cm}$ stone for a total of 2500 shocks. Intermittent fluoroscopy was performed, and it appeared there was nice fragmentation of both stones. He tolerated the procedure well without any complications. Postoperative plain film imaging demonstrated nice fragmentation of both stones (Fig. 2).

The patient returned after 2 weeks for definitive treatment with left semirigid and flexible ureteroscopy combined with laser lithotripsy for any residual fragments. Preoperative kidney, ureter, and bladder radiograph demonstrated substantial clearance of stone fragments, with small fragments potentially present in the lower pole calix and mid-ureter (Fig. 3-white arrows). With ureteroscopy, he indeed had a small cluster of small stone fragments along the mid-ureter and lower pole, which cleared easily with laser lithotripsy. Surprisingly his entire renal pelvis and upper pole and interpolar calices were completely stone free. The ureteral stent was removed 1 month later without difficulty.

\section{Discussion}

Staghorn calculi are uncommon in the pediatric population and present extremely unique challenges and difficulties in surgical treatment. Staghorn stones pose a substantial concern since the combination of infection and an increased potential for obstruction can induce damage to the renal parenchyma. ${ }^{2}$ Surgical treatment should be focused on the best

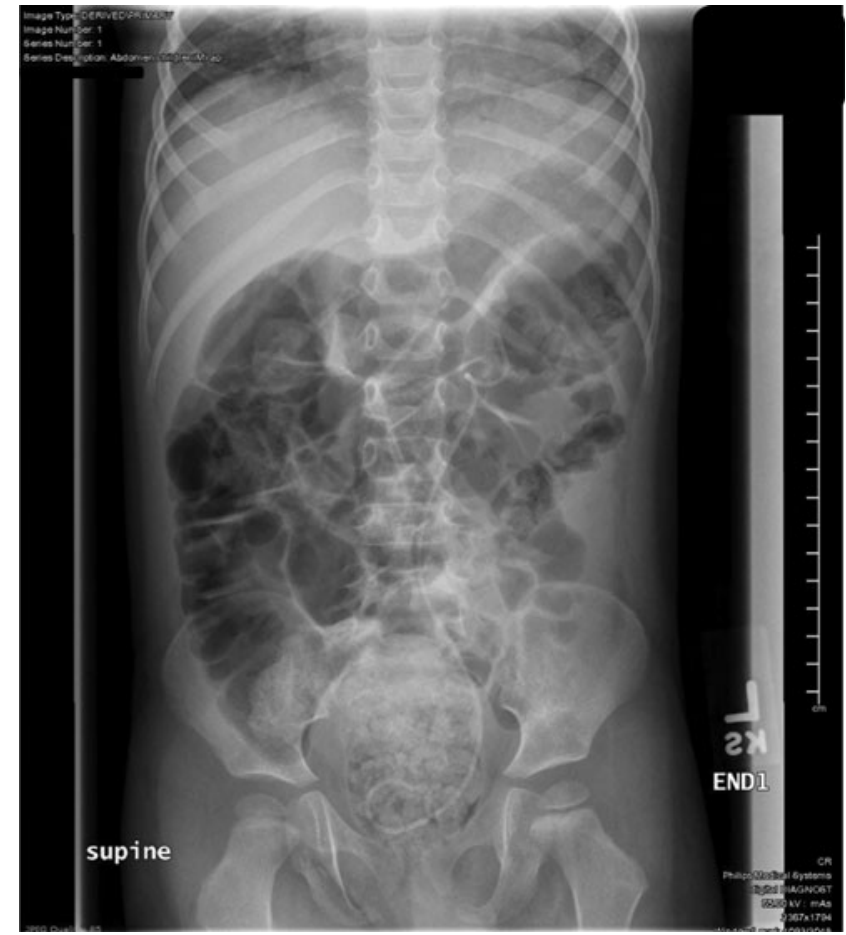

FIG. 2. Post-SWL plain film imaging demonstrating nice fragmentation of both stones. SWL = extracorporeal shockwave lithotripsy. 


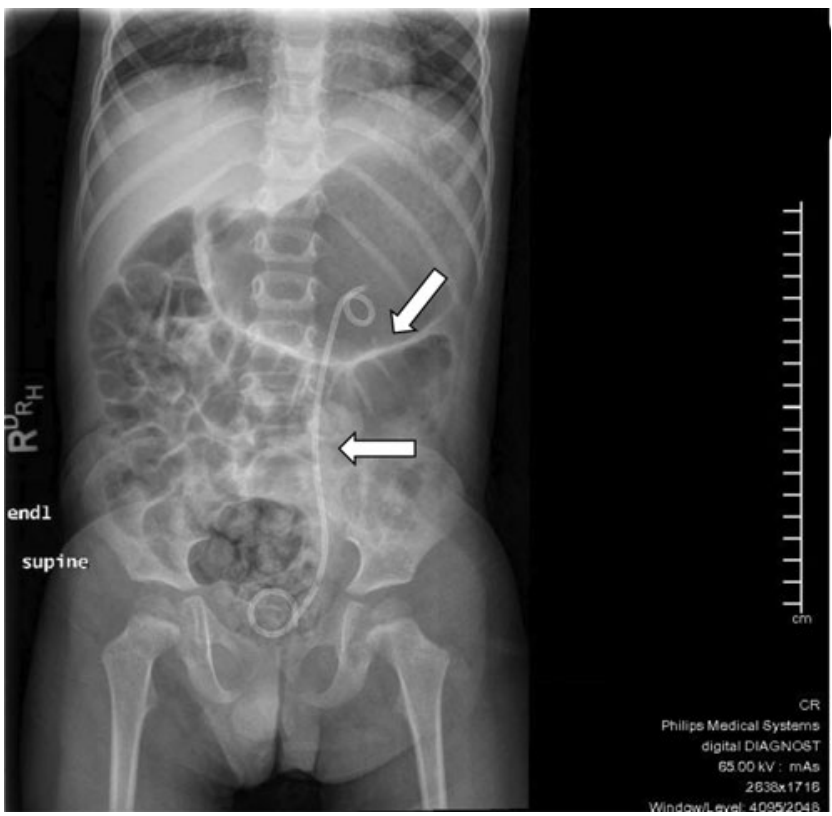

FIG. 3. Two weeks post-SWL, preoperative KUB demonstrates substantial clearance of stone fragments, with small fragments potentially present in the lower pole calix and mid-ureter (white arrows). KUB = kidney, ureter, and bladder radiograph.

modality to achieve a stone-free status. Ideally, this can be optimized with a better understanding of the stone composition, along with the different surgical approaches available. ${ }^{1}$ However, the ideal method of management for large staghorn calculi is still heavily debated.

Although monotherapy success rates have shown to be low in adults, acceptable stone-free rates in children have been achieved safely and efficiently with SWL. ${ }^{2,3}$ This is due to multiple factors, including (1) children presenting with staghorn calculi are generally struvite, or MAP, with an overall less dense and softer composition, thus easier to fragment. (2) Staghorn calculi in children are typically present in nondilated collecting systems. This poses a challenge for percutaneous nephrolithotomy approaches secondary to the limited working space in a nonhydronephrotic system. In addition, obtaining access to the upper pole stone in this patient could have been more challenging via a percutaneous approach. (3) As stated previously, a significant advantage with SWL in pediatric patients is the larger focal volume relative to the small size of this patient population. This ultimately yields more efficient fragmentation of the targeted staghorn calculus. ${ }^{2}$

While stone-free rates have been achieved using SWL, studies have demonstrated a direct relationship of worsening stone-free rates with increasing stone size. ${ }^{4}$ Few reports of $>2$-cm staghorn calculi exist in the literature detailing optimal approaches in this pediatric population. It also remains unclear to how many shocks are safe in the pediatric population. The literature reports a broad range of shocks that are efficient and safe, ranging from 900 to 3400 shocks. ${ }^{2,3}$ We have demonstrated that administering 2500 shocks to both the main staghorn and additional upper pole stone, accompanied by ureteroscopy with laser lithotripsy effectively achieved a stonefree status with high efficiency and without complications.

\section{Conclusion}

We have presented a case that demonstrates a practical treatment approach for a large staghorn calculus. Despite the numerous studies limiting the use of SWL to treat stones $<2 \mathrm{~cm}$ due to decreased stone-free rates, if used in softer composition stones, which are at times present in pediatric patients, coupled with the larger focal volume involved with smaller patients, SWL when used in combination with adjunctive ureteroscopy is a safe and effective treatment option.

\section{Disclosure Statement}

No competing financial interests exist.

\section{References}

1. Gabrielsen JS, Laciak RJ, Frank EL, et al. Pediatric urinary stone composition in the United States. J Urol 2012;187: 2182-2187.

2. Lottmann HB, Traxer O, Archambaud F, et al. Monotherapy extracorporeal shock wave lithotripsy for the treatment of staghorn calculi in children. J Urol 2001;165(6 Pt 2):23242327.

3. McLorie GA, Pugach J, Pode D, et al. Safety and efficacy of extracorporeal shock wave lithotripsy in infants. Can J Urol 2003;10:2051-2055.

4. McAdams S, Shukla AR. Pediatric extracorporeal shock wave lithotripsy: Predicting successful outcomes. Indian J Urol 2010;26:544-548.

Address correspondence to: Siam Oottamasathien, $M D$ Division of Pediatric Urology

Department of Surgery

Primary Children's Hospital

University of Utah

100 North Mario Capecchi Drive Salt Lake City, UT 84113

E-mail: siam.oottamasathien@hsc.utah.edu

\begin{aligned} & \multicolumn{1}{c|}{ Abbreviations Used } \\ & $\mathrm{CT}=$ computed tomography \\ & $\mathrm{MAP}=$ magnesium ammonium phosphate \\ & $\mathrm{SWL}=$ extracorporeal shockwave lithotripsy \end{aligned}

Cite this article as: Schults $\mathrm{AJ}$, Jia W, Ost MC, Oottamasathien S (2017) Combination extracorporeal shockwave lithotripsy and ureteroscopy for large staghorn calculi in a pediatric patient: case report, Journal of Endourology Case Reports 3:1, 64-66, DOI: 10.1089/cren.2017.0029. 